\title{
Lactobacillus salivarius
}

National Cancer Institute

\section{Source}

National Cancer Institute. Lactobacillus salivarius. NCI Thesaurus. Code C86482.

A species of facultatively anaerobic, Gram positive, rod shaped bacteria in the phylum Firmicutes. This species is nonmotile, non spore forming, nitrate negative and does not hydrolyze esculin or hippurate. L. salivarius is a commensal organism of the oral cavity. 